\title{
Der Bürger soll es richten
}

\section{Zur bürgergesellschaftlichen Durchdringung aktueller Parteiprogramme}

\author{
Peter-Georg Albrecht
}

Für Bürgerengagement sind sie alle, die großen Parteien. Was sie damit jedoch jeweils an Vorstellungen und Erwartungen verbinden, ist allerdings teilweise höchst unterschiedlich.

Alle fünf auf Bundesebene bedeutenden Parteien hatten sich mit Bundestags-Enquetekommission zur Zukunft des bürgerschaftlichen Engagements zur Jahrtausendwende auf den Weg gemacht, die deutsche Gesellschaft voranzubringen und zu reformieren. Gemeinsam wollten sie überlegen, wer im Lande eigentlich verantwortlich für (seine) Wohlfahrt ist: der Staat, der Markt und/oder der Bürger.

Nun sind sie da, die Parteiprogramme der Hinwendung zum Bürger und zur Engagementpolitik. Was bei den Grünen parallel zu den ersten Ergebnissen der von ihr als damalige Regierungspartei mitinitiierten Kommission 2002 in Berlin angedeutet wurde, hat die Linke anlässlich ihrer Fusion aus PDS und WASG im Frühjahr 2007 in Dortmund bedacht und die Sozialdemokratie im vergangenen Herbst in Hamburg detailliert durchbuchstabiert (Bündnis 90/Die Grünen. Grundsatzprogramm »Die Zukunft ist grün « vom 17.3.2002, Die Linke: Programmatische Eckpunkte vom 24./25.3.2007, SPD-Grundsatzprogramm, beschlossen am 28.9.2007).

Die mittlerweile zehnjährigen Wiesbadener Grundsätze der Liberalen heißen "Für die liberale Bürgergesellschaft « (vom 24.5.1997). Aktuell äußert sich der FDP-Bundesvorstand zum Thema Bürgergesellschaft in einem Papier über den zukünftigen liberalen Sozialmarkt (FDPBundesvorstand 2007). Die CDU/CSU,

\section{Dr. Peter-Georg Albrecht ist} wissenschaftlicher Mitarbeiter der Hochschule Magdeburg-Stendal (FH).

E-Mail peter-georg.albrecht@ hs-magdeburg.de die ihre Beiträge zum bürgergesellschaftlichen Diskurs stets zu wenig gewürdigt sieht (vgl. CDU 2007, CDU/CSU 2002: $683 \mathrm{ff}$.$) , hat kurz vor der Veröffentlichung$ des neuen sozialdemokratischen Grundsatzprogramms deutlich gemacht, dass auch sie eine treibende Kraft in der bürgergesellschaftlichen Debatte ist und dies in der Grundsatzprogrammdebatte auf dem 21. Parteitag in Hannover Anfang Dezember 2007 bestätigt.

Unter bürgergesellschaftlichen Gesichtspunkten fällt auf: Was die Programme eint, ist zuvorderst die Auffassung, irgendetwas wäre »über «, es gäbe irgendetwas zu viel. Die SPD sieht - ganz global und klassisch kapitalismuskritisch - eine $\mathrm{zu}$ große »Kluft zwischen Arm und Reich «, ein Zuviel an »Ausbeutung und Unterdrückung «. Für die CDU/CSU herrscht, ganz anders, vor allem ein $\mathrm{Zu}-$ viel an Interessengruppenmacht, an Gewerkschaften und Arbeitgeberverbänden, an politischen Gruppierungen und öffentlicher Verwaltung. Die FDP unterstreicht das Letztere: $\mathrm{Zu}$ viel Staat, zu viel Bürokratie, zu viel Umverteilung und zu viel »Gleichmacherei «, so ihre Hauptkritik. Auch die Grünen bemängeln allzu viel Staat, vor allem aber, wenn er »bevormundet « und Menschen Lasten »aufbürdet «. Die Linke macht ihre Kritik an der »Vorherrschaft der Kapitalverwertung über Wirtschaft und Gesellschaft « fest, die sich für sie in viel zu viel »chronischer Massenarbeitslosigkeit « manifestiert.

Insgesamt, so scheint es über die Kritik hinaus, geht es um Sozialpolitik, um die Konturen des Wohlfahrtsstaates. Nur: Sucht man in den Programmen nach einem wohlfahrtsgesellschaftlichen Leitbild, so fallen die Antworten sehr unterschiedlich aus. Die SPD treibt die Vision eines einerseits starken »vorsorgenden und aktivierenden ", andererseits sich aber auch zurückhaltenden Sozialstaates. Die CDU/CSU möchte so etwas wie einen zweifach subsidiären Sozialstaat. Vorrang hat für sie die Stärkung der Wettbe- werbsfähigkeit der deutschen Wirtschaft und der primären familiären Netzwerke. Zurückhaltung und Subsidiarität sind nichts für die FDP: Für sie ist ein Sozialstaat gänzlich überflüssig. Bündnis 90/Die Grünen sehen dies nicht so, sprechen aber in ihrem Programm häufig nicht konkret vom Staat, sondern von "Sozialpolitik «. Der Staat soll eine Sozialpolitik machen, die Emanzipation ermöglicht, so ihre Vision. Die Linkspartei aber will ihn selbst, den Staat, und stärker ausgeprägt noch als heute. Ihr Leitbild: ein Sozialstaat mit staatlichen Diensten.

Entsprechend dem Leitbild die Bestimmung der Verantwortlichen der Wohlfahrtsproduktion. Unterstellt man Klientelpolitik, so könnte man meinen, der Weg habe das Ziel bestimmt, aus der Bevorzugung bestimmter Akteure habe sich das wohlfahrtsstaatliche Leitbild ergeben. Für die SPD entsteht Wohlfahrt intermediär, irgendwo kooperativ zwischen Staat und Bürger und in Kooperation beider. Die CDU/CSU setzt auf klassische wertgebundene Organisationen wie beispielsweise die Kirchen. Für die Liberalen sind es Privatinitiativen und wirtschaftlich arbeitende Unternehmen, die soziale Dienstleistungen erbringen. Die Grünen präferieren Initiativen » von unten ", allerdings nicht in weniger in Gewinn abwerfender als in emanzipativer Form. Die Linkspartei sind staatliche »öffentliche Dienstleistungen « der Königsweg sozialer Wohlfahrtsproduktion.

Ein dritter Sektor von Wohlfahrtsproduzenten, jenseits von Staat und Markt, ist also ein höchst unterschiedlich definiertes Projekt. Besonders dann, wenn man den Bürger und seine primären Netzwerke dazu nimmt: Für die SPD ist der dritte Sektor ein staat- und bürgerschaftliches Kooperationsprojekt, das sich vor allem in freigemeinnützigen wie auch sozialwirtschaftlichen Unternehmungen verwirklicht. Die CDU/CSU sieht es, bei Bevorzugung konfessioneller Wohlfahrtspflege, ähnlich. 
Alle Akteure im dritten Sektor, so es ihn gibt, müssen eine starke Beziehung zum »Fundament unserer Gesellschaft «, $\mathrm{zu}$ den traditionellen Vergemeinschaftsformen von Ehe und Familie haben und diese befördern wie auch zu Engagement schaften eines ressourcenstarken Bürgers. Bündnis 90/Die Grünen konstituieren ihren dritten Sektor auch vom Bürger her. Er wird dann Realität, wenn Menschen sich selbst organisieren, artikulieren und menschenrechtliche und ökologische Ent-

\section{"Was die Programme eint, ist die Vermutung, es gäbe irgendetwas zu viel - beispielsweise zu viel >Ausbeutung oder zu viel >Gleichmacherei«"}

motivieren. Für die FDP ist ein dritter Sektor zuvorderst unabhängig, unabhängig vor allem von Staat und Tradition. Er konstituiert sich durch freies gemeinwohlbezogenes Kooperieren und Wirt-

\section{Literatur}

Alle Parteiprogrammatiken, soweit nicht anders angegeben, im Internet einsehbar. Zusatzquellen:

Bundestagsenquetekommission zur $\mathrm{Zu}-$ kunft des bürgerschaftlichen Engagements (Hg., 2002): Bericht. Bürgerschaftliches Engagement auf dem Weg in eine zukunftsfähige Bürgergesellschaft. Opladen, Leske + Budrich, S.683-727.

CDU/CSU (2002): Sondervotum von Mitgliedern der Enquete-Kommission aus der Fraktion der CDU/CSU zum Bericht der Kommission. In: Bundestagsenquetekommission zur Zukunft des bürgerschaftlichen Engagements (Hg.): S.683-727.

Dettling, W. (2007): Vom Rand in die Mitte? Perspektiven der Bürgergesellschaft. In: Forschungsjournal Neue soziale Bewegungen 2/2007, S. 7-14.

FDP-Bundesvorstand (2007): Mehr Markt im Sozialmarkt: Chance für mehr Qualität, Kosteneffizienz und Kundenzufriedenheit. Beschlusspapier vom 17.9.2007. Berlin.

Nährlich, S. (2007): Orientierungslos, mutlos, machtlos: Die Idee der Bürgergesellschaft hat es noch nicht zum gesellschaftlichen Leitbild geschafft. In: Blätter der Wohlfahrtspflege 4/2007, S. 151-152. wicklungen anstoßen. Die Linkspartei hat, so jedenfalls ihr derzeitiger Programmstand, keine explizite Vorstellung eines dritten Sektors. Der Staat hat den Kapitalismus zu domestizieren, diese Grundaufgabe kommt allen gesellschaftlichen Institutionen wie auch jedem Bürger zu.

Nur wer ist er, der Bürger, auf den sich alle Programme beziehen und den alle wollen? Kein Programm spricht vom Gleichen: Einen starken Staatsbürger wünscht sich die SPD, einen starken Familienvater die CDU/CSU, einen starken Selbstständigen die FDP, einen starken Emanzipierer Bündnis 90/Die Grünen, einen starken Kapitalismusgegner die Linkspartei (perspektivisch sollen es immer Männer sein, auch wenn die Hauptengagierten in der Gegenwart Frauen sind).

Aber ob diese doch all zu Unterschiedlichen wohl in die gleiche Richtung wollen? Es kann sie wohl nicht geben, die gemeinsame große Leitidee, nach der SPD und die Grünen zu ihrer Regierungszeit nachstrebten, und die noch heute große Programmatiker wie Dettling (2007) und Protagonisten wie Nährlich (2007) beschwören, weil es sowohl »den Bürger" als auch »das gesellschaftliche wohlfahrtsstaatliche Leitbild « nicht gibt. Es liegt also nicht "an uns selbst", lieber Stefan Nährlich, denn es gibt kein »wir «, das nur der unabhängigen Selbstorganisation harrt. Es liegt nicht an der Vision und seiner Ausstrahlungskraft, lieber Warnfried Dettling, denn zu »Selbst- und Fremdmarginalisierung « kann es nur kommen, wenn eine gewisse Einigkeit besteht.

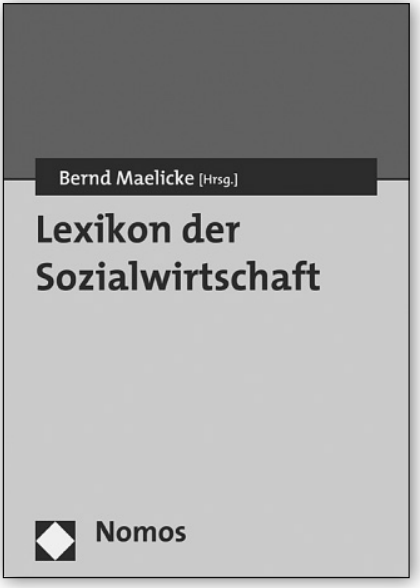

\section{Lexikon der \\ Sozialwirtschaft}

Herausgegeben von

Prof. Dr. Bernd Maelicke

2007, 1.128 S., geb., 98,-€,

ISBN 978-3-8329-2511-6

Das Werk umfasst ca. 700 Stichworte von über 130 Autoren aus den einschlägigen Fachdisziplinen Betriebswirtschaft, Volkswirtschaft, Sozialpolitik, New Public Management, Recht, Erziehungswissenschaften, Sozialarbeit/ Sozialpädagogik und Psychologie und richtet sich an die Führungskräfte in der Sozialwirtschaft/ Sozialmanagement, der Aus- und Weiterbildung sowie an die Wissenschaft.

"Es ist das erste Überblickswerk zu der Thematik und stellt auf 1.128 Seiten dar, was gegenwärtig fachlich und sachlich der Stand des Wissens zu sozialwirtschaftlichen Fragen ist."

Prof. Dr. Wolf Rainer Wendt, Zeitschrift für Betreuungsmanagement 69/08

\section{Nomos}

